\title{
Electron Energy Loss Near Edge Structures as a Tool to Elucidate Natural and Artificial Minerals Structures
}

Teresa Dennenwaldt ${ }^{1,2}$, Farhang Nabiei ${ }^{1,3}$, Duncan Alexander ${ }^{1}$, James Badro $^{3,4}$, Philippe Gillet ${ }^{3}$, Hélène Piet $^{3,5}$ and Cécile Hébert ${ }^{1,2}$

1. Interdisciplinary Center for Electron Microscopy (CIME), Ecole Polytechnique Fédérale de Lausanne, Lausanne, Switzerland

2. Electron Spectrometry and Microscopy Laboratory (LSME), Institute of Physics, Ecole Polytechnique Fédérale de Lausanne, Lausanne, Switzerland

3. Earth and Planetary Science Laboratory (EPSL), Institute of Physics, Ecole Polytechnique Fédérale de Lausanne, Lausanne, Switzerland

4. Institut de Physique du Globe de Paris, Sorbonne Paris Cité, Paris, France

5. Current address: School of Earth and Space Exploration, Arizona State University, Tempe, AZ, USA

Electron Energy Loss Near Edge Structures (ELNES) of core loss ionization edges, recorded in a TEM, are a fingerprint for the unoccupied density of states of materials. As such, they can be used to distinguish between various structures of similar composition but different electronic structure.

One example is the carbon K-edge. Its fine structure is strongly influenced by the $\mathrm{sp}^{2} / \mathrm{sp}^{3}$ hybridization of the carbon atoms, and can be used to quantify the $\mathrm{sp}^{2} / \mathrm{sp}^{3}$ ratio of materials ranging from diamond structure (pure $\mathrm{sp}^{3}$ ) to graphite structure $\left(\right.$ pure $\mathrm{sp}^{2}$ ). The main challenge lies in the required acquisition conditions to guarantee that the recorded changes are due to different hybridization and not to the anisotropy of the material. Here we show how reliable acquisition conditions can be set up even in STEM mode on a Cs corrected machine (with a priori large convergence angle of the probe) and how this allows to acquire high resolution data on a mineral samples. As an example we analyzed the Almahata Sitta 2008 TC3 asteroid [1] containing both diamond and graphite inclusions and found a clear separation between the two phases with no real mixture of the $\mathrm{sp}^{2} / \mathrm{sp}^{3}$ hybridization in the different regions of the specimens (Fig. 1).

As a second example, we show that the iron $\mathrm{L}_{2,3}$-edge can be used to determine the ratio of ferrous and ferric iron concentrations in lower mantle minerals. The lower mantle is composed of the minerals calcium perovskite $\left(\mathrm{CaPv}, \mathrm{CaSiO}_{3}\right)$, bridgmanite $\left(\mathrm{Brg},(\mathrm{Mg}, \mathrm{Fe}) \mathrm{SiO}_{3}\right)$ and ferropericlase $(\mathrm{Fp},(\mathrm{Mg}, \mathrm{Fe}) \mathrm{O})$ where the valence state and partitioning of iron have a strong impact on the physical and chemical properties [2,3]. To understand the correlation between the distribution and oxidation states of iron, samples consisting of a Brg (amorphous) and Fp (crystalline) mineral assemblage have been previously synthesized from Al-rich olivine composition at different temperatures and pressures and the $\mathrm{Fe}^{3+} / \sum \mathrm{Fe}$ has been studied with EELS (Fig. 2b and c) [4]. Things to take care of during acquisition are e.g. beam damage of the material (Fig. 2a) or electron-beam-induced oxidation of iron will be discussed.

\section{References:}

[1] P Jenniskens, et al, Nature 458 (2009), p. 485.

[2] J Badro, et al, Science 300 (2003), p. 789.

[3] C Prescher, et al, Earth and Planetary Science Letters 399 (2014), p. 86. 
[4] H Piet, et al, Proceedings of the National Academy of Sciences 113 (2016), p. 11127.
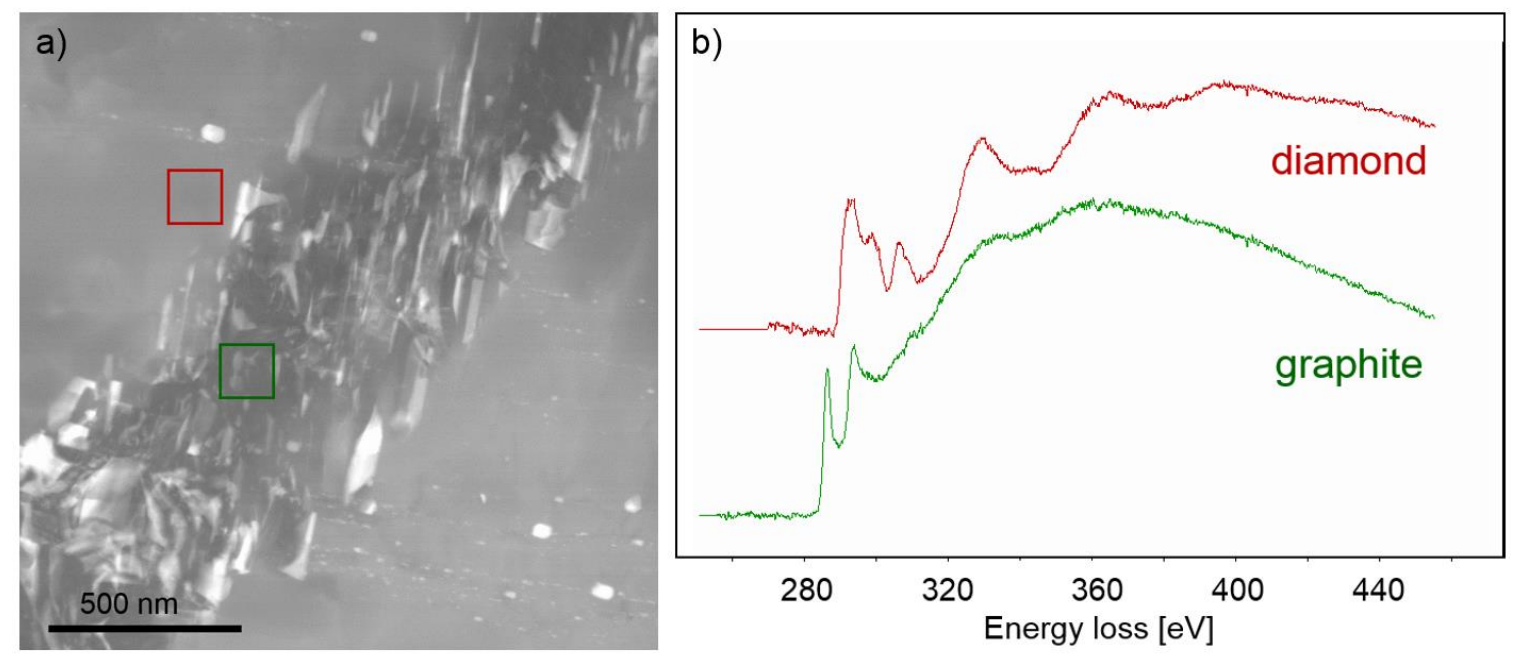

Figure 1. a) HAADF-STEM image of a meteorite sample. b) EEL spectra of the C K-edges of the diamond phase (red) and the graphite phase (green) showing a significant difference in the ELNES.
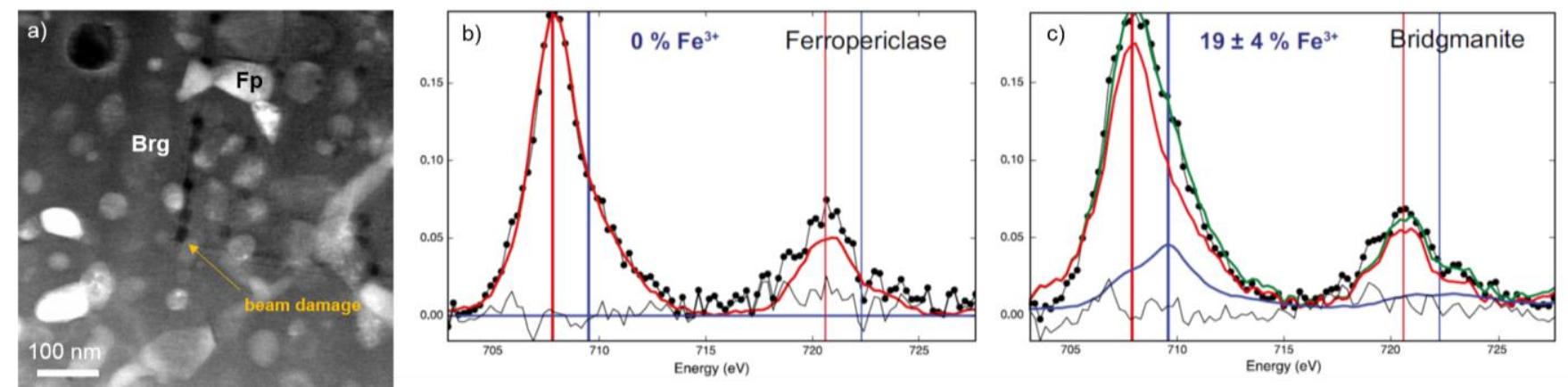

Figure 2. a) HAADF-STEM images showing beam damage, especially of the amorphous Brg matrix, b) and c) valence state analysis of the two phases Fp and Brg. The black dots are measurements on the sample, the red $\left(\mathrm{Fe}^{2+}\right)$ and blue $\left(\mathrm{Fe}^{3+}\right)$ curves are from the standards (chemical shift of $1.5 \mathrm{eV}$ ). The green curve is the calculated spectrum using the two standards. The thin black line is the residual of the fit. Fp shows no $\mathrm{Fe}^{3+}$ (b), while $\mathrm{Brg}$ has a $\mathrm{Fe}^{3+} / \Sigma \mathrm{Fe}$ value of $0.19 \pm 0.03$ (c). 